yet a lack of support can rapidly destroy this potential. In order to maintain the necessary continuity of the principal research efforts, priorities and especially posteriorities should be applied gradually, even though fast action may sometimes be required for priorities having only a short life-span.

Research councils dispose of several means for putting research priorities into practice. Depending on specific needs, the following principal instruments are employed: co-ordinated cooperation, national research programmes, international cooperation and fellowship programmes.

\section{Priority Programmes}

A study of several different priority programmes indicates that, in general, three main phases can be distinguished: preparation, decision and execution. A fourth step may consist of the evaluation and application of the results. The key question as to who finally decides on a priority programme cannot be clearly answered. Depending on the nature of the programme, the research councils or the government are competent. Not surprisingly their motives are not same. The councils are driven by scientific motives; for the government, political motives are also important. Governmental influence is usually strongest during the preparation of a programme and partly also in connection with the final decision. The other phases, especially the executive stage, are dominated by the research councils.

For most priority programmes, the time elapsing between the initiative and the final decision ranges from six months to over two years. It is essential that ways are found of reducing this relatively long timespan during the preparative phase.

A delicate matter concerns the utilization and implementation of the results obtained from priority programmes. Experience shows that the scientific conclusions do not always coincide with the views and ideas of government or parliament. Under these circumstances, mutual confidence and tolerance are indispensable.

\section{Conclusions}

In summary, the research councils and analogous bodies in western Europe are increasingly required to apply priorities in their support of research and to see that well-founded scientific contributions are made to the solution of national problems. They are the guardians of public funds and their activity has to be placed in the framework of a general national policy. Within these boundaries, however, a certain degree of autonomy and flexibility must be secured. Only then can the research councils fulfil the principal task entrusted to them, namely the support of creative basic research which is the cornerstone of industrial development and also of a nation's welfare.

\title{
Laser Induced Chemical Vapour Deposition
}

\author{
Dieter Bäuerle, Linz
}

(Angewandte Physik, Johannes Kepler University)

Laser-induced chemical vapour deposition (LCVD) is a new technique for single step local deposition or direct writing of thin films of metals, semiconductors or insulators. The lateral dimensions of these films can be varied from about $0.5 \mu \mathrm{m}$ up to several $\mathrm{mm}$. Holographic methods may permit single-step deposition of complete material patterns. In pyrolytic LCVD, the deposition rates are so high that three dimensional structures can be produced.

Flat structures with widths down to micron-size are needed in many areas of technology, such as microelectronics or integrated optics. Such structures are presently produced by standard chemical vapour deposition (CVD) together with sequential application of mechanical masking or photolithographic methods. These standard techniques, which require several production steps, are well established for large area planar substrates. On the other hand, these techniques are not very suitable for deposition of structures on nonplanar substrates. Furthermore, the increasing complexity and miniaturization of systems require controlled area coating techniques which avoid thermal or chemical cycling of entire partially fabricated devices. In such cases, LCVD is an extremely promising technique.

Applications of complex three dimensional structures produced by pyrolytic LCVD can only be speculated on. In this new and fascinating field only the first step has been taken: recently, rods of single crystalline Si have been grown within the beam of a laser - without any crucible and in an otherwise cold atmosphere. The possibility of producing non-equilibrium materials or materials which form only under extreme conditions, e.g. at high temperatures together with high pressures, is obvious.

In standard CVD, the substrate is immersed in an atmosphere of gaseous reactants in a closed reactor. The substrate is uniformly heated; the chemical reaction which leads to material deposition is thermally activated near or at the hot surface of the substrate. As a consequence, one obtains deposition of extended films.

LCVD can be based on reactions which are initiated mainly pyrolytically or photolytically, or on a combination of both.

Pyrolytic LCVD is based on local substrate heating by laser light which is not absorbed by the gaseous molecular species.
Apart from nucleation (see below), the microscopic mechanism for decomposition is the same as in conventional CVD. Therefore, the wide variety of deposition reactions used in conventional CVD can be taken over to LCVD. However, in LCVD, the chemical reaction is confined to the hot spot which is produced on the substrate or on the material deposited already - by the absorbed laser light.

The situation is quite different for photolytic LCVD. In this case the laser radiation breaks chemical bonds directly, i.e. non-thermally. Since most molecular bond energies are several $\mathrm{eV}$, ultraviolet (UV) laser light is generally required. Besides dissociative electronic excitation, dissociation of molecular species can also be achieved by multiphoton vibrational excitation with infrared laser light of suitable frequency.

\section{Surface Nucleation}

Nucleation based on purely thermal processes has been extensively studied in connection with standard thin film growth techniques. In photolytic LCVD, however, non-thermal dissociation of reactant molecules which are adsorbed at the surface of the substrate plays a basic role. This has been investigated mainly in the deposition of metal films, especially from metal-alkyls, such as $\mathrm{Cd}\left(\mathrm{CH}_{3}\right)_{2}$ and $\mathrm{Al}_{2}\left(\mathrm{CH}_{3}\right)_{6}$.

Irradiation with UV laser light of suitable frequency results in photodissociation of the adsorbed molecules, producing free metal atoms or clusters of atoms which act as nucleation centres for atoms produced in the gas phase. Moreover, there is strong evidence that, even in cases where film growth proceeds by thermal dissociation of the molecules, nucleation may be initiated by single or multiphoton dissociation of adsorbed molecules. Evidence for such processes has been obtained during $\mathrm{Cd}$ and $\mathrm{Ni}$ deposition from $\mathrm{Cd}\left(\mathrm{CH}_{3}\right)_{2}$ and $\mathrm{Ni}(\mathrm{CO})_{4}$ on transparent substrates with visible $\mathrm{Kr}^{+}$and $\mathrm{Ar}^{+}$laser radiation.

\section{Pyrolytic Deposition}

The fundamental parameter in pyrolytic deposition is the local temperature within the reaction zone. The temperature rise on a surface due to absorption of laser radiation is a function of the reflectivity and the thermal conductivity of the solid. While at the very beginning of growth these quantities are determined solely by the sub- 


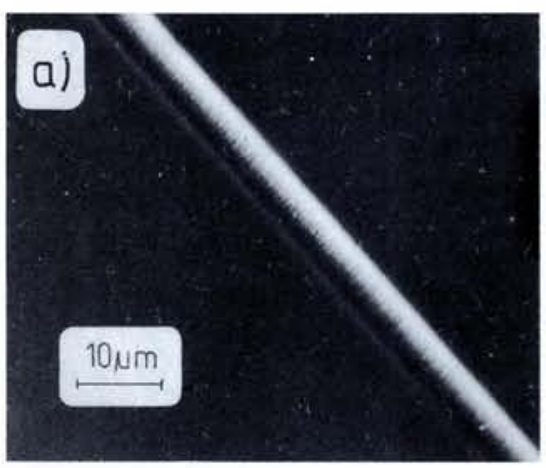

b)

Fig. 1 - a) Ni stripe grown on glass covered with $1000 \AA$ a-Si. $p\left(\mathrm{Ni}(\mathrm{CO})_{4}\right)=400 \mathrm{mbar}$, scanning velocity $v=84 \mu \mathrm{m} / \mathrm{s}, \lambda=530.9 \mathrm{~nm}, 2 w_{o}=2.5 \mu \mathrm{m}, P=6 \mathrm{~mW}$.

b) Typical thickness profile of a Ni stripe. The vertical scale is expanded ten times.

strate, they will become dependent on the deposited layer at later stages of the reaction. Because of the high deposition rates achieved in pyrolytic LCVD, one can grow structures, e.g. rods, whose vertical dimensions are so large that the deposition rate is independent of the substrate material. In the following we discuss the growth of flat structures, which is strongly influenced by the substrate, and steady growth separately.

\section{Thin Films}

Structures in the form of discs and stripes have been deposited with visible $\mathrm{Ar}^{+}$and $\mathrm{Kr}^{+}$laser radiation from various materials, such as $\mathrm{Ni}$ from $\mathrm{Ni}(\mathrm{CO})_{4}, \mathrm{Cd}$ from $\mathrm{Cd}\left(\mathrm{CH}_{3}\right)_{2}$, Si from $\mathrm{SiH}_{4}$ and from $\mathrm{Si}_{2} \mathrm{H}_{6}, \mathrm{C}$ from $\mathrm{C}_{2} \mathrm{H}_{2}, \mathrm{C}_{2} \mathrm{H}_{4}$ and from $\mathrm{C}_{2} \mathrm{H}_{6}$, and $\mathrm{SiO}_{2}$ from $\mathrm{SiH}_{4}+\mathrm{N}_{2} \mathrm{O}$. The range of laser irradiances used was $0.1-4 \mathrm{~kW} / \mathrm{mm}^{2}$; the range of partial pressures for the reactant species was from about 1 - 1000 mbar. Typical deposition rates range from some 0.1 to $100 \mu \mathrm{m} / \mathrm{s}$.

The parameters which determine the deposition of stripes are discussed below for the example of $\mathrm{Ni}$ which was deposited from $\mathrm{Ni}(\mathrm{CO})_{4}$. The results are qualitatively similar for the other systems mentioned above. Fig. 1a shows an interference contrast micrograph of a $\mathrm{Ni}$ stripe. The substrate was scanned at $84 \mu \mathrm{m} / \mathrm{s}$. The total effective laser power (measured within the reaction chamber) was $P=6 \mathrm{~mW}$ of $\lambda=$ $530.9 \mathrm{Kr}^{+}$laser radiation. A typical thickness profile of a stripe, measured interferometrically, is shown in Fig. 1b.

Both the width and the thickness of the stripes depend on laser power. Fig. 2 shows the dependence of the width on power for different substrate materials, for various laser spot diameters and for different laser wavelengths, but for constant scanning velocity and gas pressure $(v=84$ $\left.\mu \mathrm{m} / \mathrm{s}, \quad p\left(\mathrm{Ni}(\mathrm{CO})_{4}\right)=400 \mathrm{mbar}\right)$. In the cases investigated, we found that the thickness was typically a tenth to a twentieth of the width. For all but the lowest powers the width of the stripes is determined only by the physical and chemical pro- perties of the substrate (Figs. 2a-c). On the other hand, in the low power regime the growth of stripes continues to lower power for smaller diameters of the laser focus. For glass substrates, covered with a layer of $1000 \AA$ a-Si, the smallest widths of stripes achieved with a laser focus of diameter $2 w_{0}$ $=2.5 \mu \mathrm{m}$ was $1.3 \mu \mathrm{m}$ (Fig. 2a). Thus stripes can be produced which are narrower than the diffraction limit of the optical system. This increase in resolution, which is observed in the low power regime also for other diameters of the laser focus, could originate from the nonlinear dependence of the deposition rate on temperature or from a threshold irradiance necessary for nucleation.

It may be considered remarkable that deposition occurs at all on a transparent substrate such as glass (Fig. 2b) and indeed is very similar to the deposition on an absorbing substrate (Fig. 2a). This observation may be a hint that the mechanisms for nucleation are determined by local perturbations on the substrate surface (e.g. impurities or scratches) or by non-thermal processes. The latter interpretation is supported by the observation that nucleation starts more easily when the UV plasma radiation of the laser is not blocked. After nucleation, absorption seems to be determined mainly by the deposited material itself, and the overall deposition rate is for the case of $\mathrm{Ni}$ and within the accuracy of measurements - independent of laser wavelengths. As expected, the thermal conductivity of the substrate has a strong influence on the deposition rate. When using c-Si covered with a $4000 \AA$ thick thermally grown $\mathrm{SiO}_{2}$ layer instead of the glass substrate, both the width and the thickness of stripes are smaller for the same laser power.

The width and thickness of stripes decrease with increasing scanning velocity. The upper limit in the scanning velocity is determined by the break-off of stripes. For $P=24 \mathrm{~mW}, 2 w_{0}=3.4 \mu \mathrm{m}$ and a $1000 \AA$ a-Si/glass substrate, the break-off occurred at $210 \mu \mathrm{m} / \mathrm{s}$. With the higher laser power, the break-off velocity is also increased.
Pyrolytic deposition with IR light has been performed nearly exclusively with the $10.6 \mu \mathrm{m}$ radiation of pulsed or $\mathrm{CW} \mathrm{CO}_{2}$ lasers. Because the diffraction limited diameter of the laser focus is proportional to the wavelength of the light, the smallest lateral dimensions of the deposits are much larger than those achieved with visible light. For $488 \mathrm{~nm} \mathrm{Ar}{ }^{+}$and $10.6 \mu \mathrm{m} \mathrm{CO}_{2}$ laser radiation, for example, this diameter differs by about a factor of 20 (!).

\section{Steady Growth (Rods)}

Deposition rates in steady growth, W(T), can conveniently be studied quantitatively during the growth of rods (see Fig. 3). Because the deposition rate in a thermally activated process should follow an Arrhenius relation, we can write:

$W(T)=\Delta / \Delta t=W_{0} \exp (-\Delta E / k T)(2)$ where $\Delta / / \Delta t$ is the axial growing velocity of the rod, $T$ the surface temperature measured in the tip of the rod, and $\triangle E$ is an apparent chemical activation energy which characterizes the slowest step in the chain of chemical reactions involved in the deposition process. A typical example for an Arrhenius plot according to (2) is shown in the upper part of Fig. 4 for Si deposited

Fig. 2 - Dependence of width of Ni stripes on total laser power for different substrates, focus diameters and wavelengths. In all cases the total pressure was $\mathrm{p}\left(\mathrm{Ni}(\mathrm{CO})_{4}\right)=400 \mathrm{mbar}$ and the scanning velocity $v=84 \mu \mathrm{m} / \mathrm{s}$.

\section{POWER P $[\mathrm{mW}]$}

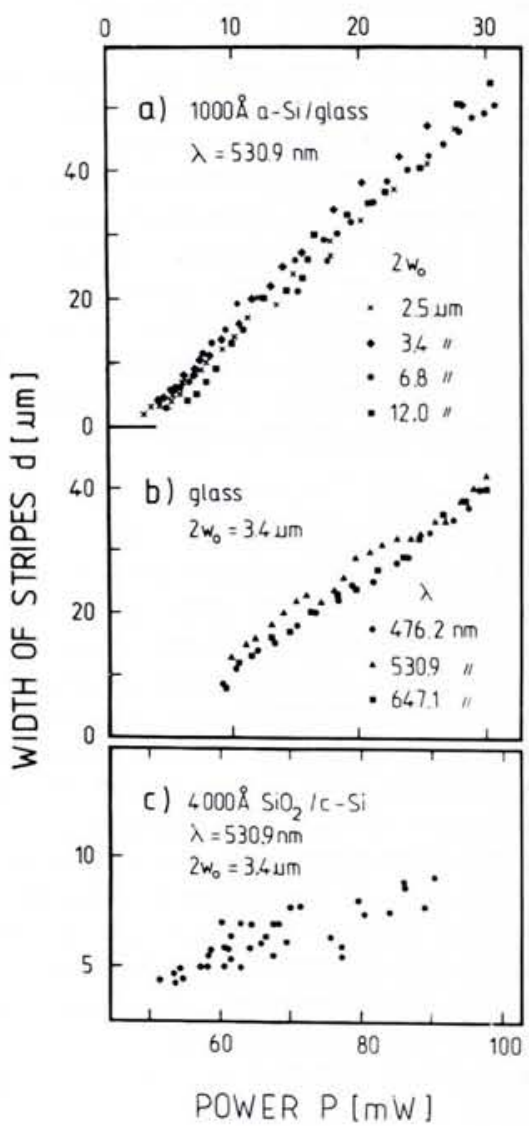




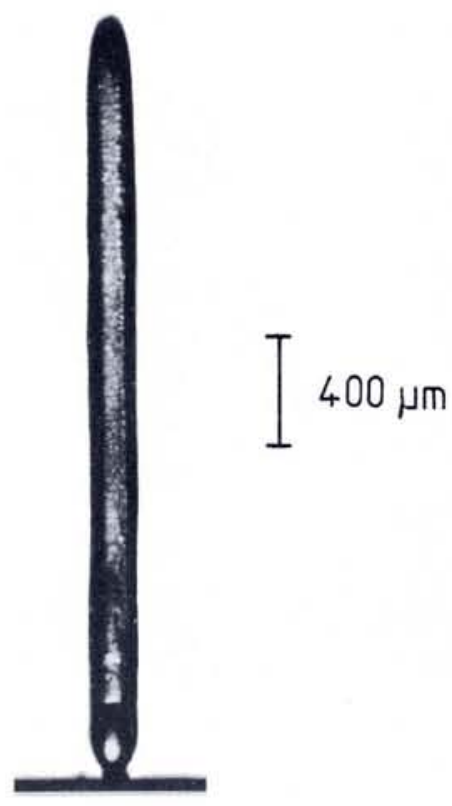

Fig. 3 - Silicon rod grown from $\mathrm{SiH}_{4}$ with 488 $\mathrm{nm} \mathrm{Ar}+$ laser radiation. $P=500 \mathrm{~mW}, \mathrm{p}\left(\mathrm{SiH}_{4}\right)$ $\approx 133$ mbar

from $\mathrm{SiH}_{4}$. Each data point marked was obtained by averaging about 30 single data points measured quasicontinuously during growth of rods along the axis of the laser beam.

Two qualitatively different temperature regimes are observed. The linear regime up to about $1400 \mathrm{~K}$ reflects an exponential increase of the deposition rate with temperature. The full line is a least squares fit to the data points in this regime; correcting its slope for the temperature dependence of the concentration of species within the reaction volume by using the ansatz $W_{0}$ $1 / T$ one obtains $\triangle E=46.6 \pm 4 \mathrm{kcal} / \mathrm{mol}$. The characteristic decrease in slope obser-

Fig. 4 - Arrhenius plots of the deposition rate in $\angle C V D$ and CVD.

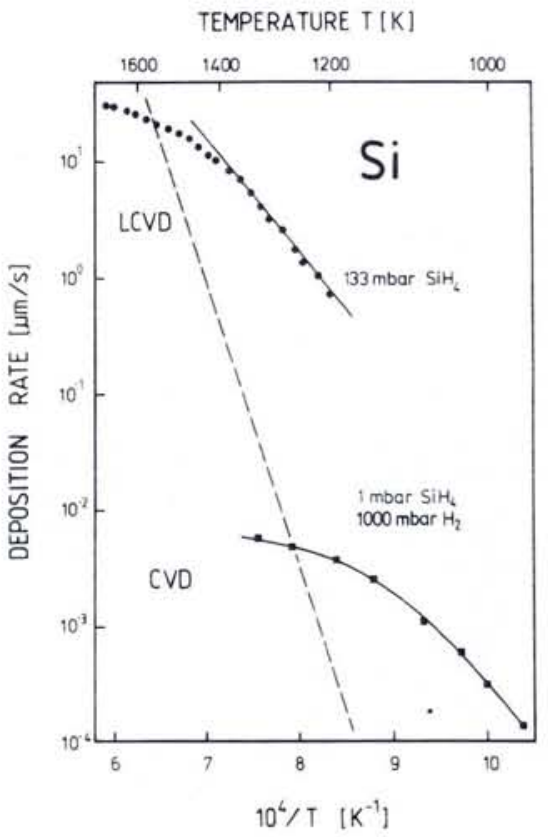

ved above a certain temperature may indicate that the decomposition process is no longer controlled by the chemical kinetics, but instead becomes limited by transport. However, alternative explanations cannot be ruled out, for example the onset of particle formation in the gas, different chemical reaction pathways, or enrichment of reaction products near the growing surface due to thermal diffusion.

The lower part of Fig. 4 shows the deposition rate for $\mathrm{Si}$ deposited from $\mathrm{SiH}_{4}-$ with $\mathrm{H}_{2}$ as carrier gas $\left(p\left(\mathrm{SiH}_{4}\right)=1 \mathrm{mbar}\right.$, $\left.p_{\text {tot }}=1000 \mathrm{mbar}\right)-$ according to standard CVD techniques.

The comparison of LCVD and CVD curves shows two remarkable differences: first, the deposition rates for LCVD are higher by a factor of $10^{2}$ to $10^{3}$; second, the kinetically controlled regime, in which the slope of the curve is independent of pressure, gas velocity, reactor geometry etc. extends to much higher temperatures. Both differences are based on the strong localization of heating in LCVD. This allows the use of much higher partial pressures of the reacting species and also higher temperatures. A further consequence of this localization of heating is the possibility of three dimensional diffusion of molecules to and from the reaction zone, while in the standard CVD large-area slab-geometry only one dimensional diffusion is effective.

\section{Photolytic Deposition}

Photodeposition by means of UV-lasers has been studied mainly for metal films produced by photolysis of the corresponding metal-alkyl and metal-carbonyl compounds. The microscopic mechanism for decomposition can be based on single photon or multiphoton processes.

Single photon decomposition was most thoroughly studied for $\mathrm{Cd}\left(\mathrm{CH}_{3}\right)_{2}, \mathrm{Zn}\left(\mathrm{CH}_{3}\right)_{2}$ and $\mathrm{Al}_{2}\left(\mathrm{CH}_{3}\right)_{6}$. The reason for the preference for these compounds is that these molecules show a dissociative continuum in the near to medium UV which can be reached by frequency doubling of a $\mathrm{CW}$ $\mathrm{Ar}^{+}$or $\mathrm{Kr}^{+}$laser. For $\mathrm{Cd}\left(\mathrm{CH}_{3}\right)_{2}$, dissociation proceeds according to the overall reaction

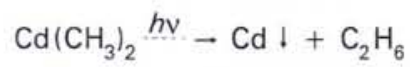

Of course, dissociation of molecules takes place within the entire volume of the laser beam. However, for thermodynamic reasons, the free gas phase atoms condense preferentially on the nuclei produced in the surface adlayer. In other words, the sticking probability for atoms or small clusters of atoms impinging on these nuclei is much larger than anywhere else on the substrate surface. Thus, isotropic deposition occurs only to a small extent. For the deposition of $\mathrm{Cd}$ from $\mathrm{Cd}\left(\mathrm{CH}_{3}\right)_{2}$ by $257.2 \mathrm{~nm}$ frequency doubled $\mathrm{Ar}^{+}$laser radiation, the deposition rate was found to increase linearly with laser irradiance as expected for single photon dissociation. For an irradiance of $1.5 \times 10^{-2} \mathrm{~W} / \mathrm{mm}^{2}, 5.3 \mathrm{mbar} \mathrm{Cd}\left(\mathrm{CH}_{3}\right)_{2}$ and zero scanning velocity, a deposition rate of about $10 \AA / \mathrm{s}$ was found. The absolute value of the deposition rate depends on a variety of parameters such as the absorption cross-section of the molecule at the laser wavelength, the partial pressure of the reactant and, when a buffer gas is used, also on its partial gas pressure. For example, at $257.2 \mathrm{~nm}$, the absorption crosssection for $\mathrm{Al}_{2}\left(\mathrm{CH}_{3}\right)_{6}$ is more than a factor of $10^{3}$ smaller than that of $\mathrm{Cd}\left(\mathrm{CH}_{3}\right)_{2}$, resulting in a corresponding decrease in deposition rate at otherwise constant conditions. This example already shows one of the main limitations for deposition based on single photon processes, because only a small number of comparably intense light sources are available at shorter wavelengths. The lateral resolution achieved in the deposition of stripes was somewhat below $1 \mu \mathrm{m}$.

Deposition of thin films $\left(\sim 5 \mathrm{~cm}^{2}\right)$ of Mo, $\mathrm{W}$ and $\mathrm{Cr}$ based on multiphoton dissociation of the hexacarbonyls was investigated with pulsed lasers such as excimer lasers, copper hollow cathode lasers, or frequency multipled Nd: YAG lasers.

Controlled growth in UV laser photodeposition was observed for a range of laser irradiances typically from $10^{-2}$ to 100 $\mathrm{W} / \mathrm{mm}^{2}$ and gas pressures in the range of about 0.1-100 mbar. Typical deposition rates achieved were 10 to some $100 \AA / \mathrm{s}$.

Photolytic LCVD induced by multiphoton absorption of IR laser radiation whose frequency matches a strong vibrational transition of the molecule has not yet been studied in much detail.

\section{Microstructure of Deposits, Single Crystals}

The microstructure of pyrolytic and photolytic deposits has been investigated mainly by optical microscopy, scanning electron microscopy (SEM), X-ray diffraction, and by Raman scattering techniques.

The microstructure of the deposits varies with experimental conditions. It can be amorphous, polycrystalline or single crystalline. The dependence of grain size on laser irradiance and gas pressure was investigated for pyrolytic deposits. Single crystal growth was first demonstrated for $\mathrm{Si}$ rods and $530.9 \mathrm{~nm} \mathrm{Kr}{ }^{+}$laser radiation. For a silane pressure of $133 \mathrm{mbar}$, the change from poly- to single- crystalline growth was observed between 1550 and $1650 \mathrm{~K}$. In this connection it is interesting to recall the microstructure of Si films grown on single crystal Si substrates by standard CVD techniques. There it has been found that the regime of polycrystalline growth is separated from the regime of single crystalline growth by a border line (shown dashed in Fig. 4), which is essentially determined by the ratio of the flux of $\mathrm{Si}$ atoms giving rise to the observed growth rate, and the 
value of the self-diffusion coefficient of $\mathrm{Si}$, needed to arrange the arriving atoms on proper lattice sites. Linear extrapolation of this border line to higher temperatures yields an intersection point with the LCVD curve at about $1520 \mathrm{~K}$. This value is in good agreement with the temperature limit we find for single-crystal growth of rods. The orientation of the rod axis was found to be close to either $\langle 100\rangle$ or $\langle 110\rangle$ crystallographic directions.

\section{Conclusion}

Laser induced deposition from the gas phase allows single-step production of material patterns with lateral dimensions from $0.5 \mu \mathrm{m}$ to several $\mathrm{mm}$. Typical deposition rates in laser pyrolysis are 10 to 100 $\mu \mathrm{m} / \mathrm{s}$ compared to 10 to some $100 \AA / \mathrm{s}$ in laser photolysis. The scanning velocities possible in laser pyrolysis reach at least up to about $500 \mu \mathrm{m} / \mathrm{s}$ for strongly adherent films. Laser pyrolysis at visible wavelengths combines high deposition rates and small lateral dimensions of deposits with standard laser techniques, simple optics and adjustment. Disadvantages of laser pyrolysis - compared to photolysis - are the stronger influence of the physical and chemical properties of the substrate and its surface quality and the higher local temperatures.

\section{BIBLIOGRAPHY}

For a description of standard chemical vapour deposition techniques see e.g.: Bloem J. and Giling L.J. in: Current Topics in Materials Science, ed. E. Kaldis (North-Holland, New York) 1978, Vol. 1, p. 147-342.

For more details on pyrolytic laser induced chemical vapour deposition see e.g. Bäuerle D. in: Laser Diagnostics and Photochemical Processing for Semiconductor Devices, eds. R.M. Osgood et al. (North Holland, New York) 1983; and Kräuter W. et al., Appl. Phys. A. 31 (1983) 13 and references therein.

Photolytic laser induced deposition is reviewed by Ehrlich D.J. et al., J. Vac. Sci. Technol. 21 (1982) 23.

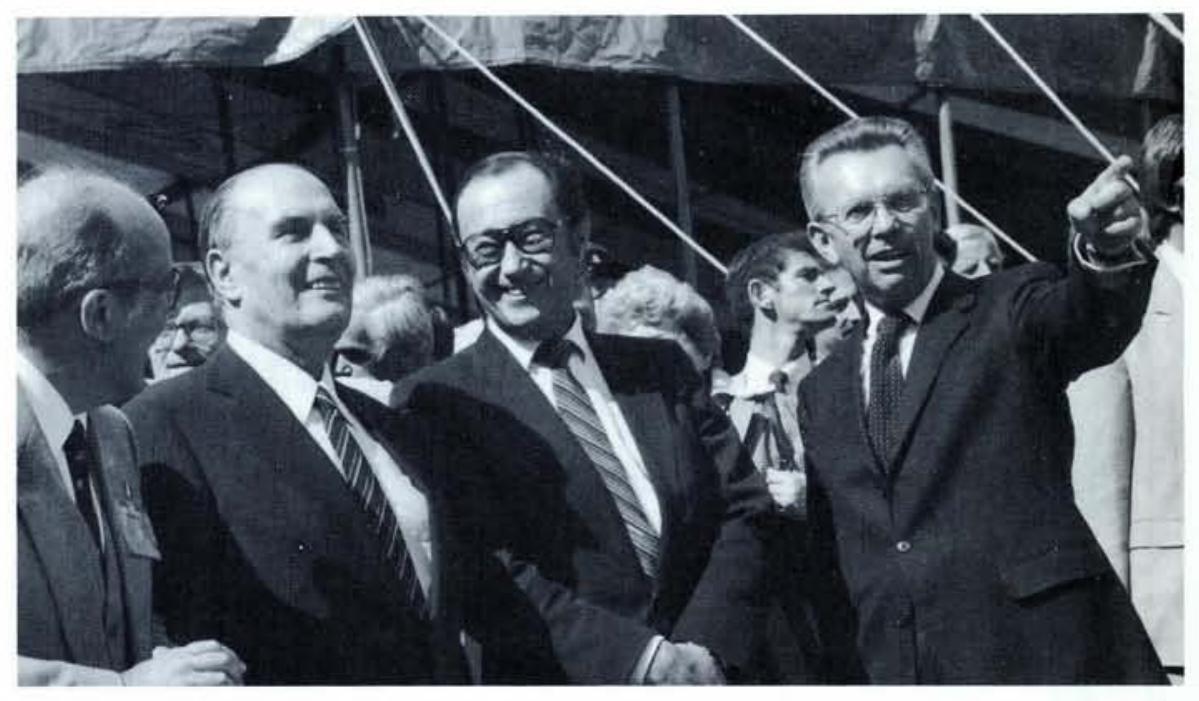

Underlining the international character of CERN, both organisational and physical, the start of construction of LEP - the 130-130 GeV electron-positron storage rings - was formally inaugurated by the Presidents of both France and Switzerland on 13 September. In the photograph taken at the ground-breaking ceremony are to the left of Francois Mitterand of France, Emilio Picasso, Director of the LEP Project and to the right of Pierre Aubert of Switzerland, Herwig Schopper, the Director-General of CERN. The $27 \mathrm{~km}$ circumference of LEP will lie 3/4 in France and 1/4 in Switzerland.

\section{UNESCO Coupons}

For the purchase of publications, films and material intended for educational, scientific or cultural purposes, buyers in some 54 countries throughout the world can make use of UNESCO Coupons. These are bought by governments in national currency from the United Nations at their official rate of exchange and are made available in the home country, sometimes with a surcharge (of $5 \%$ maximum). The coupons are redeemed by the supplier through the UNESCO Coupons Office which exacts a $3-5 \%$ handling charge that the supplier is expected to absorb. Most publishers are prepared to accept UNESCO coupons for subscription payments, as well as most equipment suppliers who are familiar with the scheme, but there is no obligation to do so.

Distributing offices in the countries covered by the EPS are as follows:
Austria:

Bulgaria :

France:

Poland:

Romania :

Spain:

UK:

Yugoslavia:

Hauptverband des Österreichischen Buchhandels Grünangergasse 4, 1010 Vienna 1

POB 386 , Sofia

CNRS, 23 rue du Maroc, 75940 Paris 19

Palac Kultury i Nauki, Warsaw

POB 71268, Bucharest

Instituto Nacional del Libro Español, Calle Santiago Rusiñol 8 , Madrid 3

Lloyds Bank International POB 241, London SW1Y 5HP

Institutes in other countries may make direct application for coupons to the UNESCO Coupons Office in Paris and this will be examined in the light of UN expenditure in the country concerned. Purchase of coupons in hard currency presents no problems.
EPS Divisions, Sections and Group

Astronomy and Astrophysics Division Solar Physics Section

Atomic and Molecular Physics Division

Atomic Spectroscopy Section

Chemical Physics

Electronic and Atomic Collisions

Molecular Physics

Computational Physics Group

Computational Physics Grou

Condensed Matter Division
Low Temperature Physics Section

Macromolecular Physics

Magnetism

Metal Physics

Semiconductors and Insulators

Surfaces and Interfaces

High Energy \& Particle Physics Division

Nuclear Physics Division

Nuclear Physics
Optics Division

Plasma Physics Division

Quantum Electronics Division
Europhysics News is the official journal of the European Physical Society which comprises 29 National Societies, Academies and Groups, over 3000 Individual Members and 68 Associate Members. Governing bodies of EPS are the General Meeting, Council and an elected Executive Committee responsible for detailed policy. EPS promotes the collaboration of physicists throughout Europe, organising and harmonising conferences, and promotes international exchanges in physics including participation in research and teaching activities abroad and attendance at schools. EPS publishes in addition to EN, Europhysics Conference Abstracts, E. Ed. News and, in collaboration with The Institute of Physics (UK), the European Journal of Physics. Individual Members receive EN free of charge (price to institutions: Sw.Fr. 82.-/a), rebates on the price of many publications and on conference fees. Annual fee for Individual Members from one of the EPS member societies for 1983 is: Sw.Fr. 40.-; independent members: SW.Fr. 120.-; APS members: Sw.Fr. 50. $-=\$ 25 .-$
Editor: E.N. Shaw

Meetings Compilation: W.S. Newman

Editorial Board:

K. Appert, A. Baratoff, B. Jacrot.

G.R. Macleod, A. Maeder, J. Muller

Editorial and Advertising Office at the EPS Secretariat.

Address: EUROPEAN PHYSICAL SOCIETY P.O. Box 69. CH-1213 Petit-Lancy 2

Switzerland

Telephone: Geneva (22) 931130

Telex: $\mathbf{4 2 3} \mathbf{4 5 5}$ dema ch

Cables: europhys genève

Printed by: Pfirter frères SA $\mathrm{CH}-1213$ Petit-Lancy/Switzerland 